\title{
JOINT SPECTRA FOR COMMUTING OPERATORS
}

\author{
by A. KÄLLSTRÖM* and B. D. SLEEMAN
}

(Received 23rd July 1984)

\section{Introduction}

The theory of joint spectra for commuting operators in a Hilbert space has recently been studied by several authors (Vasilescu $[11,12]$, Curto $[4,5]$, and Cho-Takaguchi $[2,3])$. In this paper we will use the definition by Taylor [10] of the joint spectrum to show that the joint spectrum is determined by the action of certain "Laplacians" (cf. Curto $[4,5])$ of a chain-complex of Hilbert spaces.

In particular, if $A_{1}, \ldots, A_{n}$ is a doubly commuting set of bounded linear operators, then these Laplacians are all determined by the one single operator $D=A_{1}^{*} A_{1}+\cdots$ $+A_{n}^{*} A_{n}$.

The paper is organized as follows. In Section 1 we briefly review the definition of joint spectrum. In Section 2 we discuss the role of the Laplacians in the chain-complex of Hilbert spaces described in Section 1. In Section 3 we look at the special case of doubly commuting operators and relate the spectrum of $D$ above to the joint spectrum of $A_{1}, \ldots, A_{n}$. In Section 4 we study the classification of points in the joint spectrum, particularly for the case of two commuting operators. In Section 5 we discuss an example and conjecture of Dash [7]. Finally in Section 6 the connection with the work of Vasilescu [11] is studied.

\section{Joint spectrum}

The concept of joint spectrum for commuting operators was introduced by ArensCalderon [1]. Subsequently several definitions have been given notably by Dash [6] and Taylor [10]. We will review the definition by Taylor. It is known that in certain cases the Taylor spectrum and the Dash spectrum coincide (cf. [2]).

Let $H$ be a complex Hilbert space and $A_{1}, \ldots, A_{N}$ bounded commuting linear operators in $H$. Let $e_{1}, \ldots, e_{N}$ be $N$ indeterminates and construct the exterior algebra $E^{N}$ with $e_{1}, \ldots, e_{N}$ as generators. The elements of degree $p \geqq 0$ in $E^{N}$ is the linear hull $E_{p}^{N}$ of all elements of the form

$$
e_{i_{1}} \wedge \cdots \wedge e_{i_{p}} \quad\left(1 \leqq i_{1}<\cdots<i_{p} \leqq N\right) .
$$

The space $E_{p}^{N}(H)$ is defined as $H \otimes E_{p}^{N}$, the linear hull of all the elements of the form

$$
x e_{i_{1}} \wedge \cdots \wedge e_{i_{p}} \quad\left(1 \leqq i_{1}<\cdots<i_{p} \leqq N\right) .
$$

*The research of this author was supported by SERC grant GR/C/40343 
$E_{p}^{N}(H)$ is canonically identified with a direct sum of $\left(\begin{array}{l}N \\ p\end{array}\right)$ copies of $H$ and thus it is itself a Hilbert space. We now define the maps $\delta_{p}: E_{p}^{N}(H) \rightarrow E_{p+1}^{N}(H)$ for $p=0,1, \ldots, N-1$ (where $E_{0}^{N}(H)=E_{N}^{N}(H)=H$ ) by

$$
\delta_{p}\left(x e_{i_{1}} \wedge \cdots \wedge e_{i_{p}}\right)=\sum_{k=1}^{N} A_{k} x e_{k} \wedge e_{i_{1}} \wedge \cdots \wedge e_{i_{p}}
$$

and extended by linearity. With these maps we can construct the following sequence

$$
0 \rightarrow E_{0}^{N}(H) \stackrel{\delta_{0}}{\longrightarrow} E_{1}^{N}(H) \stackrel{\delta_{1}}{\longrightarrow} \cdots \stackrel{\delta_{N-2}}{\longrightarrow} E_{N-1}^{N}(H) \stackrel{\delta_{N-1}}{\longrightarrow} E_{N}^{N}(H) \rightarrow 0 .
$$

Using the fact that the $A$ 's commute it is easily seen that (2) is a complex, i.e. that $\operatorname{im} \delta_{p} \subseteq \operatorname{ker} \delta_{p+1}$, for all $p$. This is the Koszul-complex. Furthermore, all the maps $\delta_{p}$ are bounded linear maps.

Definition 1. The $N$-tuple $A=\left(A_{1}, \ldots, A_{N}\right)$ is called non-singular if the complex (2) is exact, that is if

$$
\operatorname{im} \delta_{p}=\operatorname{ker} \delta_{p+1}, \quad p=0,1, \ldots, N-1,
$$

otherwise it is called singular.

Definition 2. The complex $N$-tuple $\lambda=\left(\lambda_{1}, \ldots, \lambda_{N}\right)$ is said to be in the joint spectrum of $A=\left(A_{1}, \ldots, A_{N}\right)$, denoted by $\sigma(A)=\sigma\left(A_{1}, \ldots, A_{N}\right)$, if $A-\lambda I=\left(A_{1}-\lambda_{1} I, \ldots, A_{N}-\lambda_{N} I\right)$ is singular.

Example 1. In the case of a single operator $A$ this reduces to the usual definition of spectrum. The Koszul-complex for this case looks like

$$
0 \rightarrow H \stackrel{A-\lambda I}{\longrightarrow} H \rightarrow 0
$$

and $A-\lambda I$ is non-singular if and only if $\operatorname{ker}(A-\lambda I)=\{0\}$ and $\operatorname{im}(A-\lambda I)=H$.

We can also define a dual complex by using the maps $\delta_{p}^{*}: E_{p+1}^{N}(H) \rightarrow E_{p}^{N}(H)$, defined by

$$
\delta_{p}^{*}\left(x e_{i_{1}} \wedge \cdots \wedge e_{i_{p+1}}\right)=\sum_{k=1}^{p+1}(-1)^{k-1} A_{i_{k}} x e_{i_{1}} \wedge \cdots \wedge \hat{e}_{i_{k}} \wedge \cdots \wedge e_{i_{p+1}}
$$

and extended by linearity. The sign $\hat{e}_{s}$ denotes omission of the factor $e_{s}$. The dual complex is

$$
0 \leftarrow E_{0}^{N}(H) \stackrel{\delta_{0}^{*}}{\longleftarrow} E_{1}^{N}(H) \stackrel{\delta_{1}^{*}}{\longleftarrow} \ldots \stackrel{\delta_{N-2}^{*}}{\longleftarrow} E_{N-1}^{N}(H) \stackrel{\delta_{N-1}^{*}}{\longleftarrow} E_{N}^{N}(H) \leftarrow 0 .
$$

It is a simple exercise to show that (2) is exact if and only if (4) is exact. This follows from the facts that $\operatorname{im} \delta_{p}$ is closed if and only if im $\delta_{p}^{*}$ is closed and that $\operatorname{ker} \delta_{p}=\left(\operatorname{im} \delta_{p}^{*}\right)^{\perp}$ $(\perp$ denoting orthogonal complement) (cf. Kato $[8]$, Theorem 5.13, p. 234). 


\section{The Laplacians of a complex}

In this section we will give a necessary and sufficient condition for a complex and its dual to be simultaneously exact at a particular point of the complex.

Consider the complex of Hilbert spaces

$$
\cdots \rightarrow H \stackrel{A}{\longrightarrow} H^{\prime} \stackrel{B}{\longrightarrow} H^{\prime \prime} \rightarrow \cdots
$$

and its dual

$$
\cdots \leftarrow H \stackrel{A^{*}}{\longleftarrow} H^{\prime} \stackrel{B^{*}}{\longleftarrow} H^{\prime \prime} \leftarrow .
$$

Here $A$ and $B$ are closed densely defined maps between the respective Hilbert spaces. This means that $A A^{*}$ and $B^{*} B$ are densely defined selfadjoint operators on $H^{\prime}$ (cf. [8], Theorem 3.24, p. 275). We assume that the "Laplacian" $D=A A^{*}+B^{*} B$ is also a closed densely defined operator. $D$ is easily seen to be symmetric and bounded from below by 0 . Hence $D$ always has a self-adjoint extension $\bar{D}_{0}$, the Friedrichs extension, with the same lower bound as $D$.

Example 2. Let us consider the case of two bounded linear operators $A_{1}$ and $A_{2}$ such that $A_{1}$ commutes with $A_{2}$ and $A_{2}^{*}$. Then also $A_{1}^{*}$ commutes with $A_{2}$ and $A_{2}^{*}$. The Koszul-complex can be written

$$
0 \rightarrow H \stackrel{\delta_{0}}{\longrightarrow} H \oplus H \stackrel{\delta_{1}}{\longrightarrow} H \rightarrow 0
$$

where $\delta_{0} x=A_{1} x \oplus A_{2} x, \delta_{1}\left(x_{1} \oplus x_{2}\right)=A_{1} x_{2}-A_{2} x_{1}$ and the dual complex

$$
0 \leftarrow H \stackrel{\delta_{0}^{*}}{\longleftarrow} H \oplus H \stackrel{\delta_{1}^{*}}{\longleftarrow} H \leftarrow 0
$$

with the maps

$$
\delta_{1}^{*} x=\left(-A_{2}^{*} x\right) \oplus A_{1}^{*} x, \delta_{0}^{*}\left(x_{1} \oplus x_{2}\right)=A_{1}^{*} x_{1}+A_{2}^{*} x_{2} .
$$

The Laplacians of the complex are

$$
\begin{aligned}
& D_{0}=\delta_{0}^{*} \delta_{0}=A_{1}^{*} A_{1}+A_{2}^{*} A_{2} \\
& D_{1}=\delta_{0} \delta_{0}^{*}+\delta_{1}^{*} \delta_{1}=\left(A_{1} A_{1}^{*}+A_{2}^{*} A_{2}\right) \oplus\left(A_{1}^{*} A_{1}+A_{2} A_{2}^{*}\right) \\
& D_{2}=\delta_{1} \delta_{1}^{*}=A_{1} A_{1}^{*}+A_{2} A_{2}^{*} .
\end{aligned}
$$

Note that if $A_{1}$ and $A_{2}$ are normal commuting operators and we put $D=A_{1}^{*} A_{1}+A_{2}^{*} A_{2}$, 
then

$$
D_{0}=D, \quad D_{1}=D \oplus D, \quad D_{2}=D .
$$

Returning now to the complexes $(C)$ and $\left(C^{*}\right)$ we state the following.

Theorem 1 (cf. [5], Prop. 3.1, p. 395). (C) and ( $\left.C^{*}\right)$ are both exact at $H^{\prime}$, (i.e. $\operatorname{im} A=$ $\left.\operatorname{ker} B, \operatorname{im} B^{*}=\operatorname{ker} A^{*}\right)$ if and only if $D$ is selfadjoint and boundedly invertible on $H^{\prime}$.

For the proof we need the following two facts about closed densely defined linear operators $T: H_{1} \rightarrow H_{2}$.

Lemma 1 (cf. [8], Theorem 5.13, p. 234). im $T$ is closed if and only if im $T^{*}$ is closed. In this case we have

$$
\operatorname{im} T=\left(\operatorname{ker} T^{*}\right)^{\perp}, \operatorname{im} T^{*}=(\operatorname{ker} T)^{\perp} .
$$

Lemma 2 (cf. [8], Theorem 5.2, p. 231), Thas closed range if and only if there is a constant $C>0$, such that

$$
\|T x\|_{2} \geqq C\|x\|_{1} \text { for all } x \in D(T) \cap(\operatorname{ker} T)^{\perp} .
$$
have

Proof of Theorem 1. First we note that since $(C)$ and $\left(C^{*}\right)$ are complexes we always

$$
\operatorname{im} A \subseteq \operatorname{ker} B, \operatorname{im} B^{*} \subseteq \operatorname{ker} A^{*} .
$$

Furthermore $\operatorname{ker} B$ and $\operatorname{ker} A^{*}$ are closed since the maps $B$ and $A^{*}$ are closed.

Assume now that $D$ is selfadjoint and $\operatorname{im} D=H^{\prime}$. Then $\operatorname{ker} D=(\operatorname{im} D)^{\perp}=\{0\}$. Hence $D^{-1}$ is a closed map defined on all of $H$ and consequently $D^{-1}$ is bounded. Now

$$
H^{\prime}=\operatorname{im} D \subseteq \operatorname{im} A+\operatorname{im} B^{*} \subseteq \operatorname{im} A+\overline{\operatorname{im} B^{*}}=\operatorname{im} A+(\operatorname{ker} B)^{\perp} \subseteq H^{\prime} .
$$

By (6) it follows that $\operatorname{im} A=\operatorname{ker} B$ and hence $(C)$ is exact at $H^{\prime}$. Similarly

$$
H^{\prime}=\operatorname{im} D \subseteq \operatorname{im} A+\operatorname{im} B^{*} \subseteq \overline{\operatorname{im} A}+\operatorname{im} B^{*}=\left(\operatorname{ker} A^{*}\right)^{\perp}+\operatorname{im} B^{*} \subseteq H^{\prime}
$$

showing that $\operatorname{im} B^{*}=\operatorname{ker} A^{*}$. Thus $\left(C^{*}\right)$ is also exact at $H^{\prime}$.

Conversely, assume that both $(C)$ and $\left(C^{*}\right)$ are exact at $H^{\prime}$. Then

$$
\begin{aligned}
\operatorname{im} A & =\operatorname{ker} B \Rightarrow \operatorname{im} A \quad \text { closed } \Rightarrow \operatorname{im} A^{*} \quad \text { closed by Lemma } 1 \\
\operatorname{im} B^{*} & =\operatorname{ker} A^{*} \Rightarrow \operatorname{im} B^{*} \quad \text { closed } \Rightarrow \operatorname{im} B \quad \text { closed by Lemma } 1 .
\end{aligned}
$$

Hence, there are constants $C_{1}, C_{2}>0$ such that

$$
\begin{gathered}
\left\|A^{*} x\right\| \geqq C_{1}\|x\| \quad x \in D\left(A^{*}\right) \cap\left(\operatorname{ker} A^{*}\right)^{\perp} \\
\|B y\| \geqq C_{2}\|y\| \quad y \in D(B) \cap(\operatorname{ker} B)^{\perp} .
\end{gathered}
$$


But any $u \in D(D)$ can be decomposed as $u=u_{1}+u_{2}$, where $u_{1} \in D(D) \cap(\operatorname{ker} B)$ and $u_{2} \in D(D) \cap(\operatorname{ker} B)^{\perp}$. But then

$$
\begin{gathered}
u_{1} \in D(D) \cap \operatorname{ker} B=D(D) \cap \operatorname{im} A=D(D) \cap\left(\operatorname{ker} A^{*}\right)^{\perp} \\
u_{2} \in D(D) \cap(\operatorname{ker} B)^{\perp}=D(D) \cap(\operatorname{im} A)^{\perp}=D(D) \cap \operatorname{ker} A^{*} .
\end{gathered}
$$

Hence $D u=A A^{*} u+B^{*} B u=A A^{*} u_{1}+B^{*} B u_{2}$ and

$$
\begin{aligned}
(D u, u) & =\left(A A^{*} u_{1}, u\right)+\left(B^{*} B u_{2}, u\right) \\
& =\left\|A_{1}^{*} u_{1}\right\|^{2}+\left\|B u_{2}\right\|^{2} \\
& \geqq C_{1}\left\|u_{1}\right\|^{2}+C_{2}\left\|u_{2}\right\|^{2} \\
& \geqq C\left\{\left\|u_{1}\right\|^{2}+\left\|u_{2}\right\|^{2}\right\} \quad C=\min \left(C_{1}, C_{2}\right)>0 \\
& =C\|u\|^{2} \quad \text { for all } \quad u \in D(D) .
\end{aligned}
$$

This shows that $\operatorname{im} D$ is closed and also that $D$ is bounded from below by $C>0$. Hence also $\bar{D}$ is bounded from below by $C>0$. But then $\operatorname{ker} \bar{D}=\{0\}$ and $\operatorname{im} \bar{D}=H^{\prime}$. But im $D$ is dense in $\operatorname{Im} \bar{D}$ and closed. Thus we must have $\operatorname{im} D=H^{\prime}$ and it follows that $D$ is already selfadjoint. One sees easily that $D^{-1}$ is bounded.

The question of the selfadjointness of $D$ can often be settled by the following.

Theorem 2 (cf. [9], p. 88). Let $G_{1}, \ldots, G_{p}$ be a finite set of selfadjoint operators such that $G_{1}, \ldots, G_{p}$ commute pairwise (i.e. their spectral families commute). In addition, suppose that $G_{i} \geqq 0, i=1, \ldots, p$. Then $G=G_{1}+\cdots+G_{p}$ is selfadjoint.

\section{Doubly commuting systems}

Let $A_{1}, \ldots, A_{N}$ be an $N$-tuple of bounded linear operators on $H$.

Definition 3. $A=\left(A_{1}, \ldots, A_{N}\right)$ is called doubly commuting if $A_{i} A_{j}=A_{j} A_{i}$ and $A_{i} A_{j}^{*}=A_{j}^{*} A_{i}$ for all $i, j=1, \ldots, N$. $A$ is called weakly doubly commuting if, for all $i=1, \ldots, N$

$$
A_{i} A_{j}=A_{j} A_{i} \text { and } A_{i} A_{j}^{*}=A_{j}^{*} A_{i} \text { for } j \neq i \text {. }
$$

In particular, in a doubly commuting system all the operators $A_{1}, \ldots, A_{N}$ are normal. The significance of these systems derive from the fact that all the Laplacians of the Koszul-complex are defined in terms of one single operator $D=A_{1}^{*} A_{1}+\cdots+A_{N}^{*} A_{N}$. The dual complex also generates exactly the same Laplacians so that the complex is in some sense "self-dual".

Let $A=\left(A_{1}, \ldots, A_{N}\right)$ be a doubly commuting system. We now want to relate the spectral subspaces of $H$ defined by the resolution of the identity belonging to $D$, to the operators $A_{1}, \ldots, A_{N}$. 
First we notice that if $\mu$ is an eigenvalue of $D$ and $E(\mu)$ is the corresponding eigenspace, then from the commutativity

$$
\begin{gathered}
(D-\mu) A_{i} x=A_{i}(D-\mu) x=0 \\
(D-\mu) A_{i}^{*} x=A_{i}^{*}(D-\mu) x=0
\end{gathered}
$$

for all $x \in E(\mu)$ and $i=1, \ldots, N$. Hence $E(\mu)$ is invariant under all $A_{i}$ and $A_{i}^{*}$. But then, if $x \in E(\mu), y \in E(\mu)^{\perp}$,

$$
\begin{aligned}
& \left(x, A_{i} y\right)=\left(A_{i}^{*} x, y\right)=0 \\
& \left(x, A_{i}^{*} y\right)=\left(A_{i} x, y\right)=0
\end{aligned}
$$

so that all the operators $A_{1}, \ldots, A_{N}, A_{1}^{*}, \ldots, A_{N}^{*}$ are reduced simultaneously by $E(\mu)$.

It is now easy to show that the discrete subspace $H_{d}$ of $H$ corresponding to the spectral resolution of $D$ is invariant under all of $A_{i}$ and $A_{i}^{*}$. Hence the continuous subspace $H_{c}$ is also invariant and all the operators $A_{1}, \ldots, A_{N}, \ldots, A_{N}^{*}$ are simultaneously reduced by $H_{d}$ and $H_{c}$. It is obvious that there are no joint eigenvalues of $A_{1}, \ldots, A_{N}$ nor of $A_{1}^{*}, \ldots, A_{N}^{*}$ in $H_{c}$.

Let us now specialize further and assume that $A_{1}, \ldots, A_{N}$ is a commuting set of selfadjoint bounded operators. Clearly, to every joint eigenvalue $\left(\lambda_{1}, \ldots, \lambda_{N}\right)$ to $\left(A_{1}, \ldots, A_{N}\right)$ there is an eigenvalue $\mu=\lambda_{1}^{2}+\cdots+\lambda_{N}^{2}$ to $D$. Conversely, if $\mu$ is an eigenvalue to $D$ and $E(\mu)$ the eigenspace, then $A_{1}, \ldots, A_{N}$ are commuting selfadjoint operators on $E(\mu)$ and hence they can be simultaneously diagonalized. In particular, if $\operatorname{dim} E(\mu)<\infty$, then there is a basis of $E(\mu)$ consisting of joint eigenvectors to $A_{1}, \ldots, A_{N}$. This leads us to the following.

Theorem 3. Suppose $A_{1}, \ldots, A_{N}$ are bounded commuting selfadjoint operators such that $D=A_{1}^{2}+\cdots+A_{N}^{2}$ is compact. Then

(i) if $\operatorname{dim} \operatorname{ker} D<\infty$ there is a complete system of joint eigenvectors of $A_{1}, \ldots, A_{N}$ in $H$.

(ii) if $\operatorname{dim} \operatorname{ker} D=\infty$ there is a complete system of joint eigenvectors to $A_{1}, \ldots, A_{N}$ in $(\operatorname{ker} D)^{\perp}$.

Proof. $D$ compact $\Rightarrow H_{c}=\{0\}$ if $\operatorname{dim} \operatorname{ker} D<\infty$, otherwise $H_{c}=\operatorname{ker} D$. Every non zero eigenvalue has finite multiplicity which means that there is a basis in the eigenspace consisting of joint eigenvectors. From this the theorem follows easily.

Remark. If $\mu$ is an eigenvalue of infinite multiplicity to $D$ there need not be any joint eigenvectors to $A_{1}, \ldots, A_{N}$ in the eigenspace as is shown by the following example.

Example 3. Let $E(\lambda)$ be a continuous spectral family. Define $A_{1}=\int \cos \lambda d E(\lambda)$, $A_{2}=\int \sin \lambda d E(\lambda)$. Then $A_{1}$ and $A_{2}$ are bounded selfadjoint commuting operators with continuous spectra only. But $D=A_{1}^{2}+A_{2}^{2}$ is the operator

$$
D=\int\left(\cos ^{2} \lambda+\sin ^{2} \lambda\right) \mathrm{dE}(\lambda)=\mathrm{I}
$$

which implies that $D$ has eigenvalue 1 with infinite multiplicity. 
In order to clarify the situation when $D$ or $D^{-1}$ is compact we prove

Theorem 4. Let $D=A_{1}^{*} A_{1}+\cdots+A_{N}^{*} A_{N}$. Then

(i) $D$ is compact if and only if all the $A_{i}$ are compact.

(ii) If at least one of the $A_{i}$ has compact inverse then $D$ has compact inverse.

Proof. (i) Assume all $A_{i}$ compact. Then trivially $D$ is compact. Conversely, if $D$ is compact, let $x_{n}$ be a sequence such that $x_{n} \rightarrow 0$ weakly. Then $D x_{n} \rightarrow 0$ strongly. But then

$$
\sum_{i=1}^{N}\left\|A_{i} x_{n}\right\|^{2}=\left(D x_{n}, x_{n}\right) \rightarrow 0 .
$$

Hence all $A_{i} x_{n} \rightarrow 0$ strongly as $n \rightarrow \infty$ which implies that all $A_{i}$ compact.

(ii) Suppose $A_{1}$ has compact inverse. Then also $A_{1}^{*}$ has compact inverse. Furthermore

$$
(D u, u)=\sum_{i=1}^{N}\left\|A_{i} u\right\|^{2} \geqq\left\|A_{1} u\right\|^{2} \geqq c\|u\|^{2} .
$$

Hence $D$ has a bounded inverse. If we now write

$$
D=A_{1}^{*} A_{1}\left(I+\left(A_{1}^{*} A_{1}\right)^{-1} \sum_{i=2}^{N} A_{i}^{*} A_{i}\right)=A_{i}^{*} A_{i}(I+C),
$$

where $C$ is a positive operator. But then $(I+C)^{-1}$ exists and

$$
D^{-1}=(I+C)^{-1} A_{1}^{-1} A_{1}^{*-1},
$$

which is compact.

\section{Classification of the spectrum}

$A=\left(A_{1}, \ldots, A_{N}\right)$ is an $N$-tuple of bounded commuting linear operators on $H$.

The following definitions are given by Dash [6].

Definition 4. $\lambda=\left(\lambda_{1}, \ldots, \lambda_{N}\right) \in \mathbb{C}^{N}$ is in the joint approximate point spectrum $\sigma_{\pi}(A)$ if there is a sequence of unit vectors $x_{n} \in H$ such that

$$
\left\|\left(A_{i}-\lambda_{i} I\right) x_{n}\right\| \rightarrow 0 \quad \text { as } \quad n \rightarrow \infty, \quad i=1, \ldots, N .
$$

Definition 5. $\lambda=\left(\lambda_{1}, \ldots, \lambda_{N}\right) \in \mathbb{C}^{N}$ is in the joint approximate compression spectrum $\sigma_{\rho}(A)$, if there is a sequence of unit vectors $x_{n} \in H$ such that

$$
\|\left(A_{i}-\lambda_{i} I^{*} x_{n} \| \rightarrow 0 \quad \text { as } \quad n \rightarrow \infty, \quad i=1, \ldots, N .\right.
$$


Let us furthermore introduce the joint point spectrum

$$
\begin{gathered}
\sigma_{p}(A)=\left\{\lambda \in \mathbb{C}^{N} ; \text { there is a non-zero } x \in H,\right. \text { such that } \\
\left.\left(A_{i}-\lambda_{i} I\right) x=0, \quad i=1, \ldots, N\right\}
\end{gathered}
$$

and the joint compression spectrum

$$
\begin{gathered}
\sigma_{\rho p}(A)=\left\{\lambda \in \mathbb{C}^{N} ; \text { there is a non-zero } x \in H,\right. \text { such that } \\
\left.\left(A_{i}-\lambda_{i} I\right)^{*} x=0, i=1, \ldots, N\right\} .
\end{gathered}
$$

If we now construct the Koszul-complex for the operators $A_{i}-\lambda_{i} I, i=1, \ldots, N$ we find easily that the Laplacians $D_{0}$ and $D_{N}$ are given by

$$
D_{0}=\sum_{i=1}^{N}\left(A_{i}-\lambda_{i} I\right) *\left(A_{i}-\lambda_{i} I\right)
$$

and

$$
D_{N}=\sum_{i=1}^{N}\left(A_{i}-\lambda_{i} I\right)\left(A_{i}-\lambda_{i} I\right)^{*}
$$

respectively. From this it is follows easily that

$$
\sigma_{p}(A) \subseteq \sigma_{\pi}(A)=\left\{\lambda \in \mathbb{C}^{N} ; D_{0} \text { not boundedly invertible }\right\}
$$

and

$$
\sigma_{\rho p}(A) \subseteq \sigma_{\rho}(A)=\left\{\lambda \in \mathbb{C}^{N} ; D_{N} \text { not boundedly invertible }\right\}
$$

We now propose to classify the spectrum according to which Laplacians are boundedly invertible.

Definition 6. The joint discrete spectrum $\sigma_{d}(A)$ is defined as

$$
\sigma_{d}(A)=\bigcup_{k=0}^{N} \sigma_{d}^{k}(A)
$$

where

$$
\sigma_{d}^{k}(A)=\left\{\lambda \in \mathbb{C}^{N} ; \operatorname{ker} D_{k} \neq\{0\}\right\}
$$

Note that the sets $\sigma_{d}^{k}(A)$ need not be disjoint.

Definition 7. The joint continuous spectrum $\sigma_{c}(A)$ is defined as

$$
\sigma_{c}(A)=\sigma(A) \backslash \sigma_{d}(A) \text {. }
$$


We decompose this in the following (not necessarily disjoint) subsets

$$
\sigma_{c}(A)=\bigcup_{k=0}^{N} \sigma_{c}^{k}(A)
$$

where

$$
\sigma_{c}^{k}(A)=\left\{\lambda \in \sigma_{c}(A) ; D_{k} \text { has an unbounded inverse }\right\}
$$

We see easily that if $A_{1}, \ldots, A_{N}$ is a doubly commuting system then

$$
\sigma_{d}(A)=\sigma_{d}^{0}(A), \quad \sigma_{c}(A)=\sigma_{c}^{0}(A)
$$

This follows since all of the Laplacians can be expressed as direct sums of $D_{0}$. Hence if $\lambda \notin \sigma_{d}^{0}(A) \cup \sigma_{c}^{0}(A), D_{0}$ is boundedly invertible and then all $D_{j}$ are boundedly invertible. Hence the complex is exact.

Example 4. Let us look at the case of a single operator. The Koszul-complex is

$$
0 \rightarrow H \stackrel{\delta_{0}}{\rightleftarrows} H \rightarrow 0
$$

$\delta_{0}^{*}$

where

$$
\delta_{0} x=(A-\lambda I) x, \quad \delta_{0}^{*} x=(A-\lambda I)^{*} x
$$

The Laplacians are

$$
\begin{aligned}
& D_{0}=(A-\lambda I)^{*}(A-\lambda I) \\
& D_{1}=(A-\lambda I)(A-\lambda I)^{*} .
\end{aligned}
$$

Hence

$$
\begin{gathered}
\sigma_{d}^{0}=\{\lambda \in \mathbb{C} ; \operatorname{ker}(A-\lambda I) \neq\{0\}\}=\text { point spectrum of } A \\
\sigma_{d}^{1}=\left\{\lambda \in \mathbb{C} ; \operatorname{ker}(A-\lambda I)^{*} \neq\{0\}\right\}=\text { compression spectrum of } A
\end{gathered}
$$

$\sigma_{\mathfrak{c}}^{0}=\{\lambda \in \mathbb{C} ; \operatorname{im}(A-\lambda I)$ dense in $H$ but not closed $\}=$ approximate point spectrum

Also $\sigma_{c}^{1}=\sigma_{c}^{0}$ since $\operatorname{im} \delta_{0}$ is not closed if and only if im $\delta_{0}^{*}$ is not closed. This is the usual decomposition of the spectrum for a single operator.

Example 5. Two commuting operators $A_{1}$ and $A_{2}$. The corresponding Koszulcomplex is (cf. Example 2)

$$
0 \rightarrow H \underset{\delta_{0}^{*}}{\stackrel{\delta_{0}}{\rightleftarrows}} H \oplus H \underset{\delta_{1}^{*}}{\stackrel{\delta_{1}}{\rightleftarrows}} H \rightarrow 0
$$


$\sigma_{d}^{0}=\left\{\lambda ; \operatorname{ker} \delta_{0} \neq\{0\}\right\}=$ joint point spectrum

$\sigma_{d}^{1}=\left\{\lambda ; \operatorname{ker} D_{1}=\operatorname{ker} \delta_{0}^{*} \cap \operatorname{ker} \delta_{1} \neq\{0\}\right\}$

$\sigma_{d}^{2}=\left\{\lambda ; \operatorname{ker} \delta_{1}^{*} \neq\{0\}\right\}=$ joint compression spectrum

$\sigma_{c}^{0}=\left\{\lambda ; \operatorname{im} \delta_{0}^{*}\right.$ not closed $\}$. But then im $\delta_{0}$ is not closed which implies that $\sigma_{c}^{0} \subseteq \sigma_{c}^{1}$.

Similarly $\sigma_{c}^{2} \subseteq \sigma_{c}^{1}$.

Conversely, if $\lambda \in \sigma_{c}^{1}$ then either $\operatorname{im} \delta_{0}$ or $\operatorname{im} \delta_{1}^{*}$ is not closed and hence $\lambda \in \sigma_{c}^{0}$ or $\lambda \in \sigma_{c}^{2}$. Hence

$$
\sigma_{c}^{1}=\sigma_{c}^{0} \cup \sigma_{c}^{2}
$$

and we get the following decomposition of the joint spectrum

$$
\sigma(A)=\sigma_{d}^{0} \cup \sigma_{d}^{1} \cup \sigma_{d}^{2} \cup \bar{\sigma}_{c}^{0} \cup \sigma_{c}^{2} .
$$

This contains the results of lemma p. 867 in [3]. have

Example 6. In case the underlying Hilbert space is finite-dimensional we clearly

$$
\sigma_{c}=\emptyset
$$

Also for the case of two operators in a finite-dimensional Euclidean space we have the relation

$$
\left.\sigma\left(A_{1}, A_{2}\right)=\sigma_{d}^{1}\left(A_{1}, A_{2}\right) \quad \text { (which implies that } \sigma_{d}^{0} \cup \sigma_{d}^{2} \subseteq \sigma_{d}^{1}\right)
$$

This can be seen as follows: $\left(\lambda_{1}, \lambda_{2}\right) \notin \sigma_{d}^{1}$ if and only if $\operatorname{ker} D_{1}=\{0\}$, equivalently $\operatorname{ker} \delta_{0}^{*} \cap \operatorname{ker} \delta_{1}=\{0\}$. Hence $\operatorname{ker} D_{1}=\{0\}$ if and only if the system

$$
\begin{array}{ll}
\left(A_{1}-\lambda_{1} I\right)^{*} x_{1}+\left(A_{2}-\lambda_{2} I\right)^{*} x_{2}=0 & \text { (i.e. } \left.\delta_{0}^{*}\left(x_{1} \oplus x_{2}\right)=0\right) \\
-\left(A_{2}-\lambda_{2} I\right) x_{1}+\left(A_{1}-\lambda_{1} I\right) x_{2}=0 & \text { (i.e. } \left.\delta_{1}\left(x_{1} \oplus x_{2}\right)=0\right)
\end{array}
$$

has only the zero solution. But then the operator

$$
\alpha(A)=\left(\begin{array}{rr}
\left(A_{1}-\lambda_{1} I\right)^{*} & \left(A_{2}-\lambda_{2} I\right)^{*} \\
-\left(A_{2}-\lambda_{2} I\right) & \left(A_{1}-\lambda_{1} I\right)
\end{array}\right)
$$

is invertible on $H \oplus H$. Hence by Theorem 1.1 in Vasilescu [11], $A-\lambda I$ is non-singular, that is $\lambda \notin \sigma(A)$.

We will here also give a proof of Theorem 3.1 in [11] based on the methods developed here. 
Let $A_{1}: H_{1} \rightarrow H_{1}$ and $A_{2}: H_{2} \rightarrow H_{2}$ be bounded linear operators with spectra $\sigma\left(A_{1}\right)$ and $\sigma\left(A_{2}\right)$ respectively. In $H=H_{1} \otimes H_{2}$ we can construct two commuting operators

$$
B_{1}=A_{1} \otimes I_{2} \quad \text { and } \quad B_{2}=I_{1} \otimes A_{2} .
$$

Let $\sigma(B)$ denote the joint spectrum of $B_{1}$ and $B_{2}$ in $H$.

Theorem 5. $\sigma(B)=\sigma\left(A_{1}\right) \times \sigma\left(A_{2}\right)$.

Proof. We have the complex

$$
0 \rightarrow H \stackrel{\delta_{0}}{\longrightarrow} H \oplus H \stackrel{\delta_{1}}{\longrightarrow} H \rightarrow 0,
$$

where $\delta_{0} x=B_{1} x \oplus B_{2} x, \delta_{1}\left(x_{1} \oplus x_{2}\right)=B_{1} x_{2}-B_{2} x_{1}$.

Note that it is enough to consider the point $(0,0)$ since

$$
B_{1}-\lambda_{1} I=\left(A_{1}-\lambda_{1} I_{1}\right) \otimes I_{2} \quad \text { and } \quad B_{2}-\lambda_{2} I=I_{1} \otimes\left(A_{2}-\lambda_{2} I_{2}\right) .
$$

The Laplacians for the complex are calculated to be

$$
\begin{gathered}
D_{0}=\left(A_{1}^{*} A_{1}\right) \otimes I_{2}+I_{1} \otimes\left(A_{2}^{*} A_{2}\right) \\
D_{1}=\left[\left(A_{1} A_{1}^{*}\right) \otimes I_{2}+I_{1} \otimes\left(A_{2}^{*} A_{2}^{*}\right)\right] \oplus\left[\left(A_{1}^{*} A_{1}\right) \otimes I_{2}+I_{1} \otimes\left(A_{2} A_{2}^{*}\right)\right] \\
D_{2}=\left(A_{1} A_{1}^{*}\right) \otimes I_{2}+I_{1} \otimes\left(A_{2} A_{2}^{*}\right) .
\end{gathered}
$$

Assume now that $(0,0) \notin \sigma\left(A_{1}\right) \cup \sigma\left(A_{2}\right)$. Then at least one of $A_{1}$ and $A_{2}$, say $A_{1}$, is non-singular, so $\operatorname{ker} A_{1}=\{0\}$ and $\operatorname{im} A_{1}=H$. But then $\operatorname{im} A_{1}$ is closed and consequently $\operatorname{im} A_{1}^{*}$ is closed and we have

$$
\operatorname{im} A_{1}^{*}=\left(\operatorname{ker} A_{1}\right)^{\perp}=H_{1}, \quad \operatorname{ker} A_{1}^{*}=\left(\operatorname{im} A_{1}\right)^{\perp}=\{0\} .
$$

Hence $A_{1}^{*}$ is also non-singular. Now $D_{0}$, being the sum of two positive operators, one of which is invertible, is boundedly invertible. Using the remark above about $A_{1}^{*}$ we can use the same kind of argument to show that $D_{1}$ and $D_{2}$ are boundedly invertible. Hence $(0,0) \notin \sigma(B)$.

Conversely, if $(0,0) \notin \sigma(B)$, then $D_{0}$ is boundedly invertible. Hence, if $w=u \otimes v$

$$
\left(D_{0} w, w\right)=\left\|A_{1} u\right\|^{2}\|v\|^{2}+\|u\|^{2}\left\|A_{2} v\right\|^{2} \geqq C\|u\|^{2}\|v\|^{2} .
$$

But then at least one of $A_{1}$ and $A_{2}$ must be boundedly invertible, for suppose $A_{2}$ is not. Then $0 \notin \sigma\left(A_{2}\right)$ and either

(i) 0 is an eigenvalue of $A_{2}$ which implies that there is a $v$ such that $\|v\|=1, A_{2} v=0$.

$\left({ }^{*}\right)$ then gives

$$
\left\|A_{1} u\right\|^{2} \geqq C\|u\|^{2} \text { which implies that } 0 \notin \sigma\left(A_{1}\right)
$$


or

(ii) 0 is in the approximate point spectrum of $A_{2}$ which implies that there is a sequence $v_{n}$ such that $\left\|v_{n}\right\|=1, A_{2} v_{n} \rightarrow 0$. But then $\left(^{*}\right)$ gives

$$
\left\|A_{1} u\right\|^{2} \geqq\left(C-\left\|A_{2} v_{n}\right\|^{2}\right)\|u\|^{2} \geqq \frac{C}{2}\|u\|^{2}
$$

if $n$ is large enough, implying $0 \notin \sigma\left(A_{1}\right)$

or

(iii) 0 is in the compression spectrum of $A_{2}$ which implies that there is a $v$ such that $\|v\|=1, A_{2}^{*} v=0$. But then from $\left({ }^{*}\right)$ on using the Laplacian $D_{2}$ instead we have

$$
\left\|A_{1}^{*} u\right\|^{2} \geqq C\|u\|^{2} .
$$

Hence $0 \notin \sigma\left(A_{1}^{*}\right)$ and so $0 \notin \sigma\left(A_{1}\right)$. Consequently if $A_{2}$ is not boundedly invertible, then $A_{1}$ must be and it follows that $(0,0) \notin \sigma\left(A_{1}\right) \times \sigma\left(A_{2}\right)$. From this follows that $\sigma(B)=\sigma\left(A_{1}\right) \times \sigma\left(A_{2}\right)$.

\section{An example by Dash}

In this section we will show that it is possible for a point $\left(\lambda_{1}, \lambda_{2}\right)$ to be in $\sigma_{d}^{1}$ but not in $\sigma_{d}^{0} \cup \sigma_{d}^{2}$. The example is given by Dash in [7] in order to disprove a certain conjecture.

Let $H=\oplus_{n=1}^{\infty} l^{2}$ so that each element $X \in H$ is a sequence of elements $X_{n} \in l^{2}, n=1,2, \ldots$. If each $X_{n}$ is given by $X_{n}=\left(x_{n 1}, x_{n 2}, \ldots\right)$ then the norm in $H$ is

$$
\|X\|^{2}=\sum_{n=1}^{\infty}\left\|X_{n}\right\|^{2}=\sum_{n, k=1}^{\infty}\left|x_{n k}\right|^{2} .
$$

Define the operators $A_{1}$ and $A_{2}$ in $H$ by the matrices

$$
A_{1}=\left(\begin{array}{cccccc}
0 & I & 0 & \ldots & \ldots & \ldots \\
0 & 0 & I & \ldots & \ldots & \ldots \\
\ldots & \ldots & \ldots & & & \\
\ldots & \ldots & \ldots & & . & \\
\ldots & \ldots & \ldots & & &
\end{array}\right) \text { and } A_{2}=\left(\begin{array}{cccccc}
V & 0 & 0 & \ldots & \ldots & \ldots \\
0 & V & 0 & \ldots & \ldots & \ldots \\
0 & 0 & V & \ldots & \ldots & \ldots \\
\ldots & \ldots & \ldots & & . & \\
\ldots & \ldots & \ldots & & & .
\end{array}\right)
$$

where $I$ is the identity operator in $l^{2}, 0$ the zero operator and $V$ the unilateral shift. Clearly $A_{1}$ and $A_{2}$ commute. Also $A_{1}$ and $A_{2}^{*}$ commute so that $A_{1}$ and $A_{2}$ form a weakly doubly commuting system.

We find for the Laplacians of the corresponding complex

$$
\begin{aligned}
\left(D_{0} f, f\right) & =\left\|A_{1} f\right\|^{2}+\left\|A_{2} f\right\|^{2} \geqq\left\|A_{2} f\right\|^{2} \\
& =\sum_{i=1}^{\infty}\left\|V f_{i}\right\|^{2}=\sum_{i=1}^{\infty}\left\|f_{i}\right\|^{2}=\|f\|^{2} .
\end{aligned}
$$


This shows that $D_{0}$ is boundedly invertible and so $(0,0) \notin \sigma_{d}^{0}$. Also

$$
\left(D_{2} f, f\right)=\left\|A_{1}^{*} f\right\|^{2}+\left\|A_{2}^{*} f\right\|^{2} \geqq\left\|A_{1}^{*} f\right\|^{2}=\|f\|^{2}
$$

which implies that $D_{2}$ is boundedly invertible and consequently

$$
(0,0) \notin \sigma_{d}^{2} .
$$

However $\operatorname{ker} D_{1} \neq\{0\}$ as the following shows:

$$
\begin{gathered}
D_{1}: H \oplus H \rightarrow H \oplus H . \text { Let } f=X \oplus Y \in \operatorname{ker} D_{1} . \text { Then } \\
A_{1} Y-A_{2} X=0 \\
A_{1}^{*} X+A_{2}^{*} Y=0 .
\end{gathered}
$$

Choose $X=0$ which implies that

$$
A_{1} Y=0 \quad \text { and } \quad A_{2}^{*} Y=0 .
$$

If $Y=\left(Y_{1}, Y_{2}, \ldots\right)$ then

$$
A_{1} Y=0 \text { if and only if } Y_{2}=Y_{3}=\cdots=0 \text { with } Y_{1} \text { arbitrary. }
$$

Now $A_{2}^{*} Y=0$ if and only if $V^{*} Y_{1}=0$ which is satisfied for $Y_{1}=\{1,0,0, \ldots\} \in l^{2}$. Hence $(0,0) \in \sigma_{d}^{1}$.

In the same paper Dash makes the following conjecture

$$
(0,0) \notin \sigma\left(A_{1}, A_{2}\right) \Leftrightarrow \text { there is an } \varepsilon>0 \text { such that }
$$

(i) $\left\|A_{1} f\right\|^{2}+\left\|A_{2} f\right\|^{2} \geqq \varepsilon\|f\|^{2}$

(ii) $\left\|A_{1}^{*} f\right\|^{2}+\left\|A_{2}^{*} f\right\|^{2} \geqq \varepsilon\|f\|^{2}$

(iii) $\left\|A_{1}^{*} f\right\|^{2}+\left\|A_{2} f\right\|^{2} \geqq \varepsilon\|f\|^{2}$

(iv) $\left\|A_{1} f\right\|^{2}+\left\|A_{2}^{*} f\right\|^{2} \geqq \varepsilon\|f\|^{2}$.

If it is easily seen that (i) holds if any only if $D_{0}$ is boundedly invertible, and that (ii) holds if and only if $D_{2}$ is boundedly invertible.

The Laplacian $D_{1}$ is in general given by a matrix operator in $H \oplus H$

$$
D_{1}=\left(\begin{array}{ll}
A_{1} A_{1}^{*}+A_{2}^{*} A_{2} & A_{1} A_{2}^{*}-A_{2}^{*} A_{1} \\
A_{2} A_{1}^{*}-A_{1}^{*} A_{2} & A_{1}^{*} A_{1}+A_{2} A_{2}^{*}
\end{array}\right)
$$

In the case of a weakly doubly commuting system the off-diagonal operators are both 
zero and we get

$$
D_{1}=\left(\begin{array}{cc}
A_{1} A_{1}^{*}+A_{2}^{*} A_{2} & 0 \\
0 & A_{1}^{*} A_{1}+A_{2} A_{2}^{*}
\end{array}\right) .
$$

For this Laplacian (iii) and (iv) hold if and only if $D_{1}$ is boundedly invertible.

Hence Dash's conjecture holds if $A_{1}, A_{2}$ are weakly doubly commuting. In the general case, however, $D_{1}$ has the following structure

$$
D_{1}=\left(\begin{array}{ll}
P & Q \\
Q^{*} & R
\end{array}\right)
$$

where $P=A_{1} A_{1}^{*}+A_{2}^{*} A_{2}, R=A_{1}^{*} A_{1}+A_{2} A_{2}^{*}$ are positive operators and $Q=A_{1} A_{2}^{*}-A_{2}^{*} A_{1}$. If $u=f \oplus g$ is a general vector in $H \oplus H, D_{1}$ is boundedly invertible if and only if there is an $\varepsilon>0$ such that $\left(D_{1} u, u\right) \geqq \varepsilon\|u\|^{2}$ or equivalently

(v) $(P f, f)+2 \operatorname{Re}(f, Q g)+(R g, g) \geqq \varepsilon\left\{\|f\|^{2}+\|g\|^{2}\right\}$.

If $g=0$ this is inequality (iii) and if $f=0$ it is inequality (iv). Hence (iii)-(iv) are necessary for $(0,0) \notin \sigma\left(A_{1}, A_{2}\right)$. However, without further conditions on the operator $Q$ it is not known whether conditions (iii)-(iv) imply (v).

\section{Connections with [11]}

In [11] Vasilescu proves the following theorem.

Theorem. $(0,0) \notin \sigma\left(A_{1}, A_{2}\right) \Leftrightarrow$ the operator

$$
\alpha(A)=\left(\begin{array}{rr}
A_{1}^{*} & A_{2}^{*} \\
-A_{2} & A_{1}
\end{array}\right)
$$

is boundedly invertible on $H \oplus H$.

We give another proof. A simple calculation shows that

$$
\alpha(A)^{*}=\left(\begin{array}{rr}
A_{1} & -A_{2}^{*} \\
A_{2} & A_{1}^{*}
\end{array}\right)
$$

and that

$$
\begin{gathered}
\alpha(A)^{*} \alpha(A)=D_{1} \\
\alpha(A) \alpha\left(A^{*}\right)=\left(\begin{array}{ll}
D_{0} & 0 \\
0 & D_{2}
\end{array}\right)
\end{gathered}
$$


where $D_{0}, D_{1}, D_{2}$ are the Laplacians of the complex for $A_{1}$ and $A_{2}$. Hence

$$
\begin{gathered}
\left(D_{1} u, u\right)=\|\alpha(A) u\|^{2} \text { and } \\
\left(D_{0} f, f\right)+\left(D_{2} g, g\right)=\left\|\alpha(A)^{*}(f \oplus g)\right\|^{2} .
\end{gathered}
$$

From this follows that if $\alpha(A)$ boundedly invertible and consequently also $\alpha(A)^{*}$. Then all of $D_{0}, D_{1}$ and $D_{2}$ are boundedly invertible which implies that $(0,0) \notin \sigma\left(A_{1}, A_{2}\right)$.

Conversely $(0,0) \notin \sigma\left(A_{1}, A_{2}\right)$ implies that all three Laplacians have bounded inverses. Hence

$$
\|\alpha(A) u\|^{2} \geqq C\|u\|^{2}
$$

which implies that $\operatorname{ker} \alpha(A)=\{0\}$ and $\operatorname{im} \alpha(A)$ is closed. Also $\operatorname{ker} \alpha(A)^{*}=\{0\}$ and hence

$$
\operatorname{im} \alpha(A)=\left(\operatorname{ker} \alpha(A)^{*}\right)^{\perp}=H .
$$

Consequently $\alpha(A)$ is boundedly invertible.

From (7) and (8) follows also an interwining property of the Laplacians. By evaluating $\alpha(A) \alpha(A)^{*} \alpha(A)$ in two different ways according to (7) and (8) we find

$$
\alpha(A) D_{1}=\left(\begin{array}{ll}
D_{0} & 0 \\
0 & D_{2}
\end{array}\right) \alpha(A) .
$$

We can also easily show the equivalence of our approach with that of Vasilescu in [12]. He defines the operators $\delta_{A}$ and $\delta_{A}^{*}$ on $\oplus_{k=0}^{N} E_{k}^{N}(H)$ by

$$
\delta_{A}=\left(\begin{array}{cccc}
0 & 0 & \ldots & \ldots \\
\delta_{0} & 0 & & \ldots \\
0 & \delta_{1} & & \ldots \\
\ldots & 0 & & 0 \\
\ldots & \ldots & \delta_{N-1} & 0
\end{array}\right), \delta_{A}^{*}=\left(\begin{array}{ccccc}
0 & \delta_{0}^{*} & \ldots & \ldots & \ldots \\
0 & 0 & \delta_{1}^{*} & & \ldots \\
\ldots & \ldots & & & \delta_{N-1}^{*} \\
0 & \ldots & & 0 & 0
\end{array}\right)
$$

and $\delta=\delta_{A}+\delta_{A}^{*}$. Clearly $\delta$ is a selfadjoint operator. The main theorem in [12] is that the complex is exact if and only if $\delta$ is boundedly invertible.

We notice that $\delta_{A}^{2}=\delta_{A}^{* 2}=0$ because of the properties $\delta_{i+1} \delta_{i}=0$ and $\delta_{i}^{*} \delta_{i+1}^{*}=0$. Hence $\delta^{2}=\delta_{A} \delta_{A}^{*}+\delta_{A}^{*} \delta_{A}$, which has the same structure as a Laplacian. A simple computation shows that

$$
\delta^{2}=\operatorname{diag}\left(D_{0}, \ldots, D_{N}\right) \quad \text { (diagonal matrix) }
$$

where $D_{0}, \ldots, D_{N}$ are the Laplacians of the complex. From this it follows immediately that $\delta$ is boundedly invertible if and only if all the Laplacians $D_{0}, \ldots, D_{N}$ are boundedly invertible.

The intertwining property (9) follows in this case by evaluating $\delta^{3}$ in two ways as

$$
\delta \operatorname{diag}\left(D_{0}, \ldots, D_{N}\right)=\operatorname{diag}\left(D_{0}, \ldots, D_{N}\right) \delta .
$$




\section{REFERENCES}

1. R. Arens and P. Calderón, Analytic functions of several Banach algebra elements, Ann. Math. 62 (1955), 204-216.

2. M. Сно and M. TAKaGUCH, Identity of Taylor's joint spectrum and Dash's joint spectrum, Stud. Math. 70 (1982), 225-229.

3. M. Cho and M. Takaguchi, Boundary of Taylor's joint spectrum for two commuting operators, Rev. Roum. Math. Pures et Appl. 27 (1982), 863-866.

4. R. E. CURTo, Fredholm and invertible $n$-tuples of operators. The deformation problem, Trans. Amer. Math. Soc. 266 (1981), 129-159. 406.

5. R. E. Curto, On the connectedness of invertible $n$-tuples, Ind. Univ. Math. J. 29 (1980), 393-

6. A. T. DaSH, Joint spectra, Stud. Math. 45 (1973), 225-237.

7. A. T. DASH, On a conjecture concerning joint spectra, J. Func. Anal. 6 (1970), 165-171.

8. T. Kato, Perturbation Theory for Linear Operators (Springer-Verlag (2nd ed.), 1976).

9. C. R. Purnam, Commutation Properties of Hilbert Space Operators and Related Topics. (Springer-Verlag, 1967).

10. J. L. TAYLOR, A joint spectrum for several commuting operators, J. Func. Anal. 6 (1970), 172-191.

11. F. H. Vasilescu, On pairs of commuting operators, Stud. Math. 62 (1978), 203-207.

12. F. H. Vasilescu, A characterization of the joint spectrum in Hilbert spaces, Rev. Roum. Math. Pures et Appl. 22 (1977), 1003-1009.

UPPSALA UNIVERSITY

Department of Mathematics

THUNBERGSVÄGEN 3

S-752 38 UpPSALA

SWEDEN
Department of Mathematical Sciences

THE UNIVERSITY

DundeE DD1 4HN

SCOTLAND, U.K. 